\title{
Editorial
}

\section{Innovación tecnológica en la gestión universitaria}

\author{
Germán Anzola Montero ${ }^{1}$
}

${ }^{1}$ Rector. Universidad de Ciencias Aplicadas y Ambientales U.D.C.A, (1Dhttps://orcid.org/0000-0001-6075-2595

Cómo citar: Anzola Montero, G. 2019. Innovación tecnológica en la gestión universitaria. Rev. U.D.C.A Act. \& Div. Cient. $22(2): e 1380$. http://doi.org/10.31910/rudca.v22.n2.2019.1380

Artículo de acceso abierto publicado por Revista U.D.C.A Actualidad \& Divulgación Científica, bajo una licencia Creative Commons CC BY-NC 4.0

Estudios y experiencias señalan que la innovación no es espontánea como otras acciones que suceden en la naturaleza y la sociedad, pero sí existen metodologías y estrategias que mejoran la probabilidad de generar ideas innovadoras, siendo el caso, de la adecuada utilización de la tecnología para lograr mejores resultados en los procesos y las actividades que se desarrollan en la vida diaria de las personas y las instituciones.

Siendo conscientes de los retos y las oportunidades que trae la cuarta revolución industrial, en donde los más beneficiados serán los capaces de adaptarse e innovar, eficientemente, existe la necesidad de ser más recursivos y creativos, en la forma como se utilizan los recursos tecnológicos existentes, con el fin de impactar, de manera más efectiva, en el alcance de las aspiraciones y de los objetivos.

Haciendo un rápido resumen de los principales avances tecnológicos de las denominadas revoluciones industriales es posible comprender los factores más relevantes de cada una de ellas y cómo fueron modificando las necesidades de innovación y de adaptación a los cambios que, cada una, conllevó.

La primera revolución industrial, en el siglo XVIII, estuvo determinada por la innovación en métodos de producción, con la utilización del hierro y el carbón, generando el combustible, que permitió los avances y el impacto en la productividad con la máquina a vapor. La segunda revolución industrial, siglo XIX, logró gran impacto, en la manera de hacer las cosas, con nuevas formas de generación de energía, como fueron la electricidad y el uso del petróleo, abriendo el camino para que, en el siglo XX, con la obtención masiva de energía y el fortalecimiento de las comunicaciones, se lograran avances tecnológicos, como el Internet, la fibra óptica y la nanotecnología, llegando a lo que se denomina como la tercera revolución industrial, que estuvo enmarcada por la necesidad de trabajar multidisciplinarmente en I + D + I (Investigación, Desarrollo e Innovación).

La cuarta revolución industrial, siglo XXI, plantea una nueva forma de organizar los procesos, con la utilización de diversas tecnologías, como la inteligencia artificial, la robótica, el internet de las cosas, la interconexión de múltiples dispositivos y con máquinas que hacen máquinas.

Una de las características de la cuarta revolución industrial es que no cambiará lo que hacemos, sino cómo lo hacemos, modificando las variables de lugar y de tiempo, por la disponibilidad de la conectividad y el acceso a diversos dispositivos en todo momento, lo cual, invita a una amplia reflexión sobre las oportunidades con la aparición de nuevas profesiones, transformando la mano de obra hacia personas que requieran capacidades digitales, la posibilidad de analizar grandes cantidades de datos y la forma de compartir el conocimiento. "Estamos al borde de una revolución tecnológica que modificará 
fundamentalmente la forma en que vivimos, trabajamos y nos relacionamos. En su escala, alcance y complejidad, la transformación será distinta a cualquier cosa que el género humano haya experimentado antes", vaticina Klaus Schwab (2016), fundador del Foro Económico Mundial y autor del libro "La cuarta revolución industrial". En la figura 1, publicada por el World Energy Trade, se puede apreciar la evolución de las eras industriales.
En Colombia y particularmente en el sector educativo, los rectores que representan a las Instituciones de Educación Superior que conforman la Asociación Colombiana de Universidades, ASCÚN, no han sido ajenos al análisis de la realidad que afronta hoy el mundo, en cuanto a la transformación de las profesiones y la necesidad de innovar en la forma de transmitir el conocimiento a la nueva

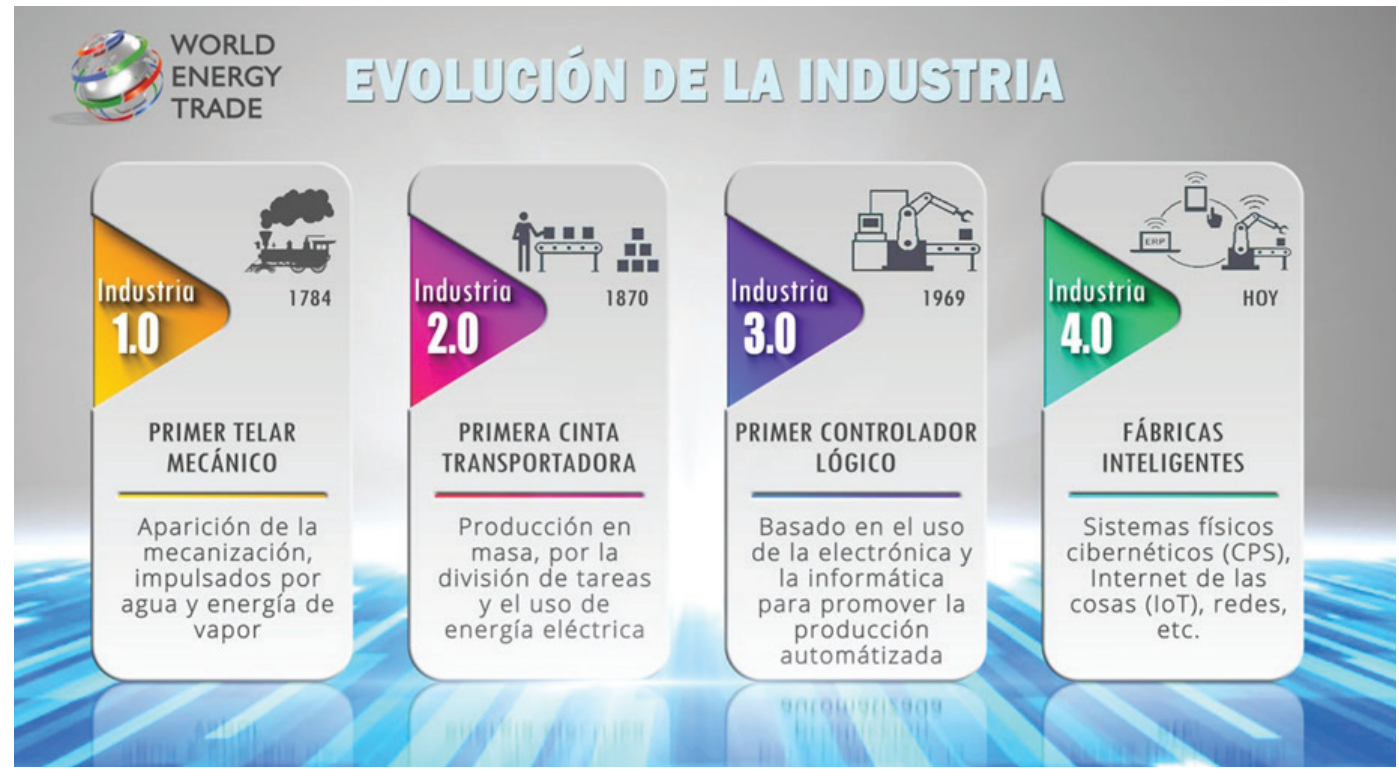

Figura 1. Industria 4.0: la necesidad de gestionar la transición para afrontar el impacto de las nuevas tecnologías. Imagen tomada de: World Energy Trade (2019).

generación de estudiantes, tema analizado en diferentes foros y encuentros universitarios, identificando puntos de reflexión, para incorporar al interior de las universidades, como son:

- Un mundo donde las competencias se reevalúan permanentemente y los modelos de negocio se transforman.

- Una disrupción digital, que requiere transformación en las capacidades para ser más competitivos y prever ambientes simulados.

- Las organizaciones están reentrenando el recurso humano en alianza con las universidades e, incluso, algunas están desarrollando sus propias escuelas de formación.

- Las empresas ya no piden, como requisito, profesiones sino capacidades, a partir de competencias específicas, en cortos tiempos de preparación.

- La tendencia de las universidades es ofrecer experiencias conectadas, investigación sin límites, aprendizajes de por vida.

- Los trabajos están cambiando y se debe propiciar el acceso a todos a la tecnología para reducir la brecha digital.

- Los estudiantes asumen, al presente, un papel activo en la renovación de empresas y se deben propiciar proyectos multidisciplinares compartidos con las empresas.

- Oferta de posibilidades de aprendizaje fuera del aula.

- Cambio del rol del docente.
- Las empresas no encuentran egresados con capacidad de cuestionarse y de innovar.

- La planeación curricular debe tener muy en cuenta el desarrollo de competencias socioemocionales y estar acorde con el contexto de problemáticas reales.

El vicepresidente de la Universidad Laurea de Ciencias Aplicadas, en Finlandia, el Doctor Kyösti Väkeväinen (2019), plantea, como puntos para considerar al interior de las universidades para mitigar el impacto de los cambios actuales, trabajar, en el corto plazo, en el desarrollo de competencias pedagógicas en los profesores; en el mediano plazo, en las políticas digitales de las universidades y el desarrollo curricular, acorde a cada una de las disciplinas en que se están formando a los estudiantes y, en el largo plazo, en la planeación estratégica de las universidades, enfocándose en las necesidades de el con quién y el para quién se ofrecen los programas académicos, se realiza la investigación y se soporta la proyección social, como razón de ser de las instituciones, en procura de una mejor sociedad.

La Universidad de Ciencias Aplicadas y Ambientales U.D.C.A, siendo consecuente con la realidad de transformación en el mundo, viene trabajando, de manera permanente, desde hace 36 años, en el proceso de autoevaluación y búsqueda de la excelencia, al servicio 
de la comunidad académica nacional e internacional, en las áreas de conocimiento que, por vocación impacta, con el fin de mantenerse vigente y actualizada, para afrontar los retos que se presentan en la sociedad; por lo cual, no solo está en continua revisión curricular de sus programas, en la formación de los profesores en pedagogías y utilización de nuevas tecnologías, que permiten estar a la vanguardia para la transmisión de conocimientos y acompañamiento a sus estudiantes. En lo referente a la calidad de la gestión universitaria, el uso de la infraestructura informática y la utilización de sistemas de información, se han gestionado inversiones que propenden por optimizar y apoyar la dinámica de los procesos misionales de la Universidad, la óptima administración de los recursos institucionales, la planeación académica, la implementación de herramientas para el acompañamiento virtual del aprendizaje, la gestión documental y la comunicación, para que los estudiantes y los profesores puedan estar conectados e informados de las actividades de la vida universitaria, los avances en la ruta académica y el cumplimiento del calendario académico.

La transformación digital e innovación en la gestión académica y de la investigación en la Universidad, se basa en el sistema de información UNIVERSITAS XXI, un sistema de gestión integral para universidades, desarrollado por la Oficina de Cooperación Universitaria Española (OCU), que opera en más de cien Instituciones, en España y en América Latina.

El sistema cubre los procesos misionales en la gestión de una institución de educación superior: gestión de programas académicos, proceso de admisión, configuración de la programación de cursos, gestión de la matrícula financiera, gestión de la matrícula académica, evaluación docente, proceso de calificaciones, gestión de los controles de la normatividad académica institucional, automatizar el proceso de grado y la gestión de la investigación, temas destacados de la vida universitaria, que fueron analizados y discutidos en el Foro de Usuarios de UNIVERSITAS XXI, que convocó en septiembre de 2019, en Madrid, España, a más de 50 universidades del mundo, con la participación de la U.D.C.A, representada por la Rectoría, en donde, durante las jornadas académicas, se presentaron los avances en las tendencias de transformación digital, con desarrollo del software, utilizando metodologías ágiles, blockchain, soluciones Cloud y la experiencia de usuario, con el objetivo de optimizar los procesos académicos de las Universidades e impactar, de manera positiva, sus niveles de productividad e innovación.

La U.D.C.A tiene como aliado estratégico a esta reconocida empresa europea, líder en soluciones tecnológicas, que viene implementando los módulos de este sistema de información con gran éxito, permitiéndole agilizar y optimizar la labor que adelantan los macroprocesos de Formación, de Extensión y Proyección Social, así como de Investigación y Gestión del Conocimiento, puestos al servicio de directivos, de profesores y de estudiantes, mediante el procesamiento y la disposición de la información, lo que permite la moderna gestión académica, administrativa y la oportuna toma de decisiones. UNIVERSITAS XXI incorpora herramientas para la difusión y el análisis de la información gestionada y herramientas para la integración con otros sistemas de gestión, como los que, actualmente, se tienen con el sistema financiero SEVEN y el sistema de Recursos humanos Kactus, desarrollados por la empresa Digital

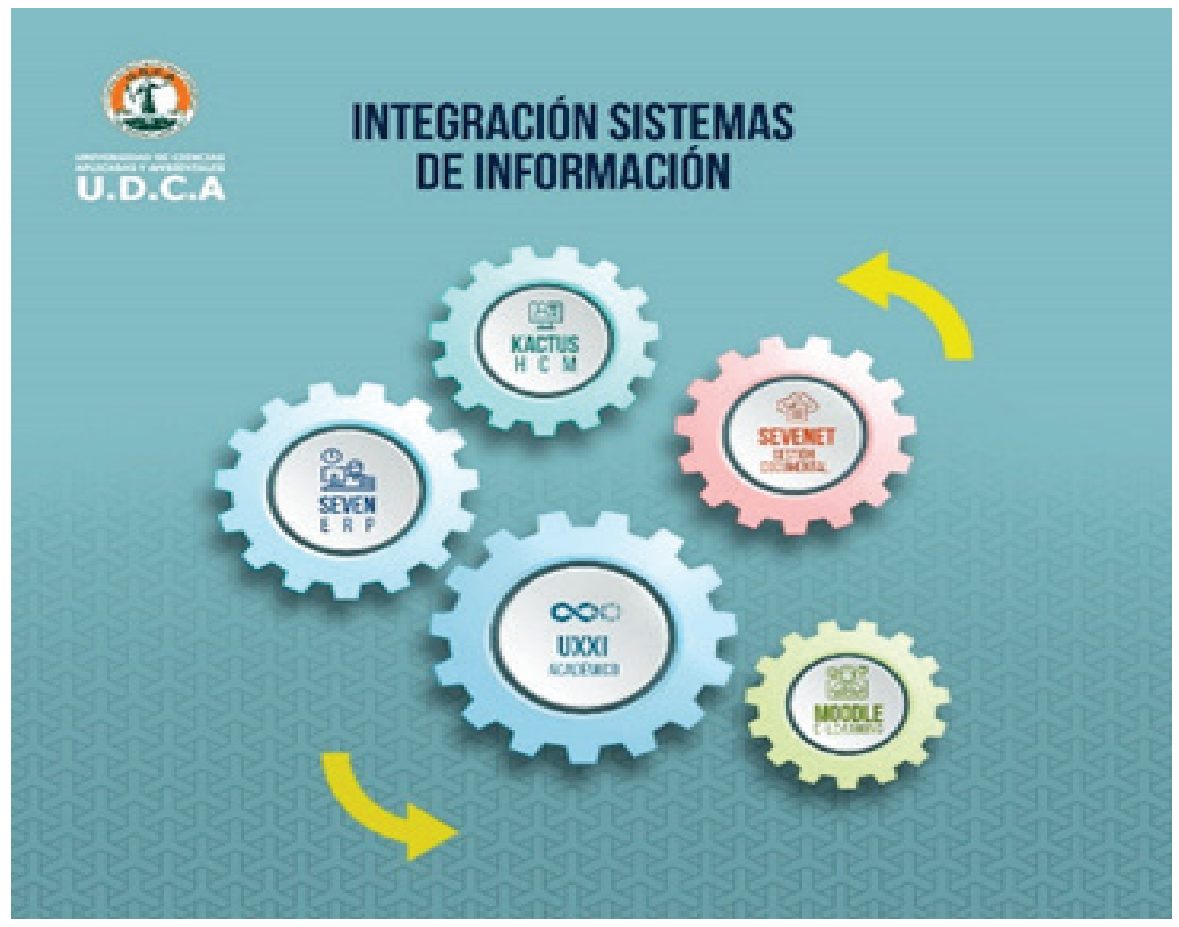

Figura 2. Principales sistemas de información integrados en la Universidad de Ciencias Aplicadas y Ambientales. Esquema elaborado por la Gestión de Tecnologías de la Información y la Comunicación. 
Ware, así como, la plataforma para el apoyo de la educación virtual Moodle y el sistema de información de Gestión Documental, Sevenet de la empresa Lexco (Figura 2).

El sistema de información financiero SEVEN está orientado a gestionar, eficientemente, las necesidades en las áreas administrativas, financiera y comerciales, que permite procesar información en tiempo real, haciendo más fácil la toma de decisiones en la U.D.C.A. Este software está integrado con el Sistema de Talento Humano KACTUS-HR y con el Sistema Académico UNIVERSITAS XXI, para los procesos de admisión y matrículas de los estudiantes. La gestión del talento humano, siendo estratégica, contempla los módulos necesarios para la administración de los procesos de convocatoria, de selección, de contratación y de nómina de los funcionarios y profesores de la Universidad. Con el fin de sistematizar los requerimientos de gestión documental en forma integral, la Universidad cuenta con un sistema de información documental SEVENET, que permite digitalizar documentos, como también clasificar los archivos por medio de carpetas digitales, facilitando, con ello, consultar y recuperar información, de manera ágil y eficiente. Adicionalmente, habilita la creación de flujos de trabajo para la comunicación institucional y el trámite de documentos hacia el interior y exterior de la U.D.C.A, impactando de la misma forma, en la gestión de la retención documental, acorde con las políticas que ayudan a controlar el ciclo de vida de la información, la disminución del uso de papel y el cumplimiento con la normatividad vigente en Colombia (Ley 594 de 2000).

Por último, se destaca, como proyecto académico de innovación enmarcado en las políticas institucionales, las directrices y las orientaciones rectorales, encaminadas a la trasformación de la calidad académica de la Universidad, a través de la innovación educativa con la utilización de las TIC para la docencia, que facilita la flexibilización curricular y la diversificaron de la oferta formativa a diferentes públicos y audiencias en los diversos contextos, a nivel local, regional y nacional. El proyecto institucional denominado PlanTIC, se definió con el propósito de desarrollar capacidad institucional para el uso de tecnología, como factor transformador de la docencia y el fortalecimiento de los procesos didácticos y de aprendizaje, mediados por las TIC, generando, junto con los profesores, ambientes virtuales, como apoyo a la presencialidad y acompañamiento al trabajo independiente de los estudiantes, mediante metodologías de E-Learning y B-Learning, aplicando las herramientas ofrecidas por MOODLE, que buscan, con la generación de Objetos Virtuales de Aprendizaje (OVA), que los estudiantes puedan acceder, desde cualquier dispositivo y lugar, a los cursos ofrecidos en pregrado, posgrado y extensión.

En la Visión de la U.D.C.A, declarada en su Proyecto Educativo Institucional, PEI, se evidencian elementos clave, que orientan los planteamientos estratégicos para la incorporación de las TIC en los procesos de innovación académicos y administrativos, en tanto, se propone: "ser reconocida en el contexto local, regional, nacional e internacional, como una Universidad que se distingue por su excelencia académica y su compromiso con la responsabilidad ambiental, puesta al servicio del desarrollo bumano sostenible".

\section{REFERENCIAS}

1. CONGRESO DE COLOMBIA. 2000. LEY 594 DE 2000. (Julio 14). "Por medio de la cual se dicta la Ley General de Archivos y se dictan otras disposiciones". Disponible desde Internet en: https://www.mintic.gov.co/portal/604/ articles-15049_documento.pdf (con acceso 25/10/2019).

2. SCHWAB, K. 2016. La cuarta revolución industrial. Ed. Debate, España, 224p.

3. VÄKEVÄINEN, K. 2019. Panel educación superior y cuarta revolución industrial. Asociación Colombiana de Universidades ASCÚN. Disponible desde Internet en: https://ascun.org.co/uploads/default/ news/216ee0676efd7b06722b6d4cdab2340a.pdf (con acceso 25/10/2019).

4. WORLD ENERGY TRADE. 2019. Industria 4.0: la necesidad de gestionar la transición para afrontar el impacto de las nuevas tecnologías. Disponible desde Internet en: https://www.worldenergytrade.com/index. $\mathrm{php} / \mathrm{m}$-articulos-tecnicos/187-news-articulos-tecnicosenergias-alternativas/3841-industria-4-0-la-necesidad-degestionar-la-transicion-para-afrontar-el-impacto-de-lasnuevas-tecnologias (con acceso el 25/10/2019). 